\title{
The effect of anthocyanin supplementation in modulating platelet function in sedentary population: a randomised, double-blind, placebo-controlled, cross-over trial
}

\author{
Kiara Thompson ${ }^{1}$, Holly Hosking ${ }^{2}$, Wayne Pederick ${ }^{2,3}$, Indu Singh ${ }^{4}$ and Abishek B. Santhakumar ${ }^{1 *}$ \\ ${ }^{1}$ School of Biomedical Sciences, Australian Research Council (ARC) Industrial Transformation Training Centre for Functional \\ Grains, Charles Sturt University, Wagga Wagga, NSW 2678, Australia \\ ${ }^{2}$ School of Medical and Applied Sciences, Central Queensland University, Rockhampton, QLD 4701, Australia \\ ${ }^{3}$ QML Pathology, Rockhampton, QLD 4700, Australia \\ ${ }^{4}$ School of Medical Science, Griffith University, Gold Coast, QLD 4215, Australia
}

(Submitted 15 June 2017 - Accepted 23 July 2017)

\begin{abstract}
The anti-thrombotic properties of anthocyanin (ACN) supplementation was evaluated in this randomised, double-blind, placebo (PBO) controlled, cross-over design, dietary intervention trial in sedentary population. In all, sixteen participants (three males and thirteen females) consumed $\mathrm{ACN}(320 \mathrm{mg} / \mathrm{d})$ or PBO capsules for $28 \mathrm{~d}$ followed by a 2 -week wash-out period. Biomarkers of thrombogenesis and platelet activation induced by ADP; platelet aggregation induced by ADP, collagen and arachidonic acid; biochemical, lipid, inflammatory and coagulation profile were evaluated before and after supplementation. ACN supplementation reduced monocyte-platelet aggregate formation by $39 \%$; inhibited platelet endothelial cell adhesion molecule-1 expression by $14 \%$; reduced platelet activation-dependant conformational change and degranulation by reducing procaspase activating compound-1 (PAC-1) ( $\downarrow 10 \%)$ and P-selectin expression ( $\downarrow 14 \%)$, respectively; and reduced ADP-induced whole blood platelet aggregation by $29 \%$. Arachidonic acid and collagen-induced platelet aggregation; biochemical, lipid, inflammatory and coagulation parameters did not change post-ACN supplementation. PBO treatment did not have an effect on the parameters tested. The findings suggest that dietary ACN supplementation has the potential to alleviate biomarkers of thrombogenesis, platelet hyperactivation and hyper-aggregation in sedentary population.
\end{abstract}

Key words: Anthocyanins: Platelet function: Thrombosis: CVD

One of the primary contributors to vascular dysfunction and pro-thrombotic progression in CVD is due to hyperactivation and hyper-aggregation of platelets ${ }^{(1)}$. Lack of physical activity has been proposed as an independent cardiovascular risk factor $^{(2)}$. Sedentary lifestyle is defined as an individual who performs less than $3 \mathrm{~h}$ of aerobic exercise per week ${ }^{(3)}$. However, during irregular acute exercise or absence of physical activity, an increase in free radical mediated oxidative stress and pro-thrombotic progression has been observed ${ }^{(2,4)}$. During increased oxidative stress and endothelial vessel wall damage in such pro-thrombotic conditions, platelets adhere, activate and aggregate at the site of injury ${ }^{(5)}$. Consequently, platelet activation targets activation-dependent surface receptors: $\mathrm{P}_{2} \mathrm{Y}_{1} / \mathrm{P}_{2} \mathrm{Y}_{12}$ (ADP receptor), GPVI/ $\alpha_{2} \beta_{1}$ (collagen receptor); and thrombotic pathways: cyclo-oxygenase-1 (COX-1) arachidonic acid pathway $^{(6)}$. During endothelial vessel wall damage and platelet activation, leucocytes and primarily monocytes form aggregates with platelets at the site of injury ${ }^{(7,8)}$. This mechanism of leucocyte adhesion to activated platelets is an important contributor to atherosclerotic plaque development in a high oxidative stress environment $^{(7)}$ such as in sedentary individuals ${ }^{(9)}$. Current antiplatelet therapeutics are designed to blunt such specific thrombotic pathways thereby reducing platelet activation and the subsequent risk of $\mathrm{CVD}^{(6,10)}$. Though current anti-platelet drugs demonstrate platelet activation and aggregation inhibiting properties, there have been reports on increased resistance and side effects in target populations ${ }^{(11)}$. Research focusing on natural dietary antioxidants and polyphenols, with anti-platelet benefits has been increasingly investigated ${ }^{(1,12-15)}$. Recent evidence suggests polyphenols and their subclasses are effective in reducing CVD risk factors by demonstrating anti-hypertensive effects, endothelial function improvement and blocking of specific platelet pathways, therefore inhibiting platelet activation and aggregation $^{(16)}$. Anthocyanins (ACN), a subclass of the polyphenol family, flavonoids, have demonstrated the ability to reduce the risk of thrombosis via its free radical scavenging and

Abbreviations: ACN, anthocyanins; PAC-1, procaspase activating compound-1; PBO, placebo.

* Corresponding author: Dr A. B. Santhakumar, email asanthakumar@csu.edu.au; sabishekbommannan@gmail.com 
metal chelating properties ${ }^{(17,18)}$. Recent in vitro and in vivo studies have shown ACN-rich foods to improve endothelial function ${ }^{(19)}$, decrease lipid peroxidation ${ }^{(20)}$, demonstrate potent antioxidant capabilities ${ }^{(21)}$ and reduce inflammatory markers ${ }^{(22,23)}$. This study aims to investigate the potential of 4-week ACN capsule supplementation in inhibiting specific mechanistic pathways of thrombogenesis in sedentary pro-thrombotic individuals.

\section{Methods \\ Study participants and experimental design}

This study was performed in compliance with the guideline laid down in the Declaration of Helsinki and was approved by the Central Queensland University Human Research Ethics Committee, QLD, Australia (approval no: H15/07-154). All participants provided informed consent before the commencement of the study. This study was also registered with the Australian New Zealand Clinical Trials Registry (ACTRN12615000797572). In all, sixteen sedentary participants (three males and thirteen females) with a BMI of $<25 \mathrm{~kg} / \mathrm{m}^{2}$ were recruited from the local community. Screening via interviews and questionnaires was conducted to ensure participants were sedentary, healthy, nonsmokers, were not taking any anti-inflammatory, antiplatelet medications or health supplements and had no history of metabolic or CVD. The definition of sedentary population has been established as participants who perform less than $3 \mathrm{~h}$ of aerobic exercise per week ${ }^{(3,9)}$. Standard dietary intake and antioxidant questionnaires were conducted and reviewed before testing to ensure participants involved in the study did not consume a diet high in antioxidants. A randomised, doubleblind, placebo (PBO) controlled, cross-over design was applied. Baseline fasting blood samples were collected from all participants to evaluate any pre-existing abnormalities in full blood count parameters, biochemical analysis, platelet activity and aggregation, oxidative stress and inflammatory markers as well as overall haemostatic function. Blood pressure and anthropometric measurements were also recorded to determine participant's BMI. Upon initial screening, participants were grouped into $\mathrm{A}$ and $\mathrm{B}$ and supplemented with $\mathrm{ACN}$ extract or PBO capsules accordingly. An external body that worked independently to the study investigators randomly assigned participants to each group. Supplementation began after baseline testing, for $28 \mathrm{~d}$, where they consumed two capsules in the morning and two capsules in the afternoon per day ( $80 \mathrm{mg}$ each) of either ACN or PBO. Capsule intake and compliance were monitored upon return of the capsule strips after each supplementation bout. After $28 \mathrm{~d}$ of supplementation (either ACN or PBO), blood pressure, body measurements and blood testing were repeated on day 29 followed by a 2 -week wash-out period, then supplementation cross-over was performed and analysis repeated, that is on days 43 and 71 . Due to the rapid absorption and elimination of most anthocyanins, the 2 -week wash-out period was deemed sufficient to avoid carryover ${ }^{(24)}$. This wash-out period was utilised by our group in a previous study evaluating the consumption of ACN-rich Queen Garnet plum juice on platelet activation-related thrombogenesis in healthy volunteers $^{(25)}$.

\section{Blood sample collection}

Blood was collected at each visit from the median cubital vein by a trained phlebotomist using a 21-gauge butterfly needle into, two tri-potassium EDTA $(1.8 \mathrm{mg} / \mathrm{ml})$ anticoagulant tube for full blood count analysis, four tri-sodium citrate $(28 \cdot 12 \mathrm{~g} / \mathrm{l}$ concentration) tubes for platelet aggregation, surface marker expression and coagulation assays, and serum separation tube (SST) for biochemical analysis. Careful handling of citrate tubes was ensured to minimise agitation and to prevent any artifactual platelet activation. SST tubes were left standing upright for $>30 \mathrm{~min}$ to clot then centrifuged at $3000 \mathrm{rpm}$ for $10 \mathrm{~min}$. Samples were visually inspected for clots, and none were obtained from traumatic blood collection.

\section{Supplementation capsules}

The ACN supplements were produced by Medapalett Pharmaceuticals, Biolink. The supplements consisted of a hemicellulose capsule containing purified ACN extract $(80 \mathrm{mg}$ ) from wild Norwegian bilberries (Vaccinium myrtillus) and black currants (Ribes nigrum) - ACN capsules contained $33.0 \%$ of 3-O- $\beta$-glucosides, 3-O- $\beta$-galactosides and 3-O- $\beta$-arabinosides of cyanidin; $58.0 \%$ of $3-O-\beta$-glucosides, $3-O-\beta$-galactosides and $3-O-\beta$-arabinosides of delphinidin; $2.5 \%$ of $3-O-\beta$-glucosides, 3-O- $\beta$-galactosides and $3-O-\beta$-arabinosides of petunidin; $2.5 \%$ of 3 - $O$ - $\beta$-glucosides, 3 - $O$ - $\beta$-galactosides and $3-O-\beta$-arabinosides of peonidin; $3.0 \%$ of 3 - $O$ - $\beta$-glucosides, $3-O-\beta$-galactosides and 3-O- $\beta$-arabinosides of malvidin; and $1.0 \%$ of 3 - $O$-rutinoside of cyanidin and delphinidin. In addition, the ACN capsules also contained pullulan, maltodextrin, and citric acid (which took up $4 \%$ per capsule for the stability of the ACN). The PBO capsules were composed of maltodextrin and a blue colour additive which contained no phenolic compounds and appeared identical to ACN capsules. ACN supplementation of $320 \mathrm{mg} / \mathrm{d}$ has been shown as an effective treatment amount in previous studies owing to its bioavailability.

\section{Dietary intake and physical activity monitoring}

Upon commencement of the study, participants were instructed not to make any adjustments to their usual diet. In order to monitor diet and physical activity during their supplementation periods, participants were required to record $24 \mathrm{~h}$ full food intake of: (1) type of food eaten, (2) preparation involved, (3) amount and (4) time, once a week throughout each period of their 4-week supplementation bout ( 8 weeks in total). Records of dietary intake (food diary) were monitored using FoodWorks $^{\circledR}$ (Xyris Software Pty Ltd) based on the Australian Food Composition database. The monitoring process also consisted of a physical activity log, a record of which was required to ensure participants performed less than $3 \mathrm{~h}$ of exercise per week and maintained a sedentary lifestyle.

\section{Biochemical profile}

Biochemical analysis including electrolytes, inflammatory markers, liver/kidney function, oxidative stress markers and lipid profiles were performed on a Beckman Coulter AU680 spectrophotometry and potentiometry biochemistry analyser 
(Beckman Coulter Inc.) with colorimetry, turbidimetry, latex agglutination, homogenous enzyme immunoassay and indirect ion selective electrode analytical capability methods. Troubleshooting, reagent management and machine maintenance were performed before testing along with quality control to ensure accurate analyser capability.

\section{Full blood count and coagulation profile}

Full blood count analysis was performed using a Sysmex XT-1800i (Sysmex Canada, Inc.) haematology analyser to evaluate general haematological parameters such as; $\mathrm{Hb}$, haematocrit, erythrocyte count, leucocyte count, platelet count, mean platelet volume and platelet distribution width. A Sysmex CA-600 (Siemens Healthineers) coagulation analyser was used for coagulation profile analysis consisting of prothrombin clotting time, activated partial thromboplastin time, fibrinogen concentration and fibrinogen degradation product (FDP)/ innovance D-dimer via clot detection. Tri-sodium citrate tubes were centrifuged at $3000 \mathrm{rpm}$ for $10 \mathrm{~min}$ to produce platelet poor plasma used for coagulation profile testing.

\section{Biomarkers of thrombogenesis and platelet activation}

The analysis was performed and interpreted using a BD FACVerse ${ }^{\mathrm{TM}}$ flow cytometer (BD Biosciences). Platelet activity and thrombogenic indicators were assessed via activation-dependent platelet monoclonal antibodies and their respective isotype controls. Procaspase activating compound-1 (PAC-1)-fluorescein isothiocyanate-fluorescein isothiocyanate was used to detect platelet activation related conformational change, P-selectin/ CD62P-allophycocyanin-allophycocyanin highlighted activation dependent de-granulation, CD42b-phycoerythrin/CD14-phycoerythrin-cyanin 7 expression determined monocyte-platelet aggregate formation and platelet endothelial cell adhesion molecule-1 (PECAM-1)/CD31-allophycocyanin-cyanine 7 expression represented cellular adhesion. Monocyte-platelet aggregates were expressed as percentage parent (\%), and activated platelets were expressed as mean fluorescence intensity. A decreased expression of $\mathrm{mAb}$ exhibits alleviation of thrombogenesis. Within $5 \mathrm{~min}$ of collection tri-sodium citrated whole blood was used for assay preparation

to avoid artifactual activation of platelets. A mixture of all monoclonal antibodies $(16 \mu \mathrm{l})$ was added to $50 \mu \mathrm{l}$ of whole blood and incubated for $15 \mathrm{~min}$ at room temperature in the dark. To induce platelet activation, ADP $(1.65 \mu \mathrm{l})$ was added, and samples were incubated for a further $15 \mathrm{~min}$ in the dark at room temperature, after which erythrocytes were lysed $(532 \mu \mathrm{l}$ of $10 \%$ lysing solution). Samples were thoroughly vortexed to ensure homogeneity and incubated in the dark at room temperature for a further $10 \mathrm{~min}$ and then analysed. In all, 10000 platelet events were acquired, gated on the basis of light scatter and CD42b mAb expression.

\section{Platelet aggregation}

Whole blood platelet aggregation studies were performed on a Chrono-log model 700 (DKSH Australia Pty. Ltd) aggregometer and analysed via electrical impedance $(\Omega)$ to evaluate platelet aggregation occurring in the sample over a 6-min period. Citrated whole blood was stimulated with ADP $(10 \mu \mathrm{m}-10 \mu \mathrm{l})$ $30 \mathrm{~min}$ after collection, collagen $(1 \mu \mathrm{g} / \mathrm{ml}-1 \mu \mathrm{l})$ and arachidonic acid $(0.5 \mathrm{~mm}-10 \mu \mathrm{l})$. A quantity of $500 \mu \mathrm{l}$ of blood and $500 \mu \mathrm{l}$ saline were incubated for $5 \mathrm{~min}$, the sample cuvette was then analysed by inserting the probe, setting the baseline and once stability was maintained, the platelet agonist (ADP, collagen or arachidonic acid) was added. All samples were analysed in duplicates.

\section{Statistical analysis}

GraphPad Prism version 7.0 for Mac OS X (GraphPad Software) was used to analyse all data. A repeated-measures ANOVA followed by Newman-Keuls post hoc multiple comparisons was carried out. A minimum sample size of fourteen subjects in each group was required for $80 \%$ power to detect a $5 \%$ variation in the laboratory parameters (platelet aggregation) measured, where a $3-5 \%$ sD exists in the population, assuming an alpha error of $0 \cdot 05$. All data has been expressed as a means and standard deviations. Differences between the groups were considered significant when the value for $P<0 \cdot 05$. Any significant statistical interactions were included in the analysis where applicable.

\section{Results}

The baseline parameters of the participants in the study were all within normal reference ranges as established by the Royal College of Pathologists of Australasia ${ }^{(26)}$. There were no significant changes to the full blood counts; coagulation profile including plasma fibrinogen concentration and FDP (D-dimer) (Table 1); biochemical and inflammatory markers following ACN or PBO supplementation (online Supplementary Table S1). Assessment of physical activity records demonstrated that participants did not perform more than $3 \mathrm{~h}$ of exercise per week throughout the study (data not shown). Evaluation of the food intake did not demonstrate any variation in the micronutrients or macronutrient levels across the two supplementation bouts (Table 2). The results are presented in the form of homogenised data (males and females) and there were no significant differences between the two populations.

\section{Biomarkers of thrombogenesis and platelet activation}

ACN supplementation alleviated thrombotic progression and endothelial dysfunction associated leucocyte migration/ adhesion by demonstrating a reduction in ADP-induced monocyte-platelet aggregate formation (Fig. 1) and PECAM-1 expression (Fig. 2) by 39 and $14 \%$, respectively. Initial platelet activation associated conformational change represented by PAC-1 expression (Fig. 3) and activation-dependent platelet degranulation represented by P-selectin expression (Fig. 4) was also reduced by 10 and $14 \%$, respectively post-ACN supplementation. PBO supplementation did not affect thrombogenic biomarkers or platelet activation. 
Table 1. Effect of anthocyanin (ACN) and placebo (PBO) supplementation on full blood count and coagulation profile (Mean values and standard deviations)

\begin{tabular}{|c|c|c|c|c|c|c|c|c|c|}
\hline \multirow[b]{2}{*}{ Parameters } & \multicolumn{2}{|c|}{$\mathrm{PRE}_{\mathrm{ACN}}$} & \multicolumn{2}{|c|}{$\mathrm{POST}_{\mathrm{ACN}}$} & \multicolumn{2}{|c|}{$\mathrm{PRE}_{\mathrm{PBO}}$} & \multicolumn{2}{|c|}{$\mathrm{POST}_{\mathrm{PBO}}$} & \multirow[b]{2}{*}{ Reference range } \\
\hline & Mean & SD & Mean & SD & Mean & SD & Mean & SD & \\
\hline Age (years) & \multicolumn{9}{|c|}{$38(12)$} \\
\hline Height (m) & 1.67 & 0.09 & 1.67 & 0.09 & 1.67 & 0.09 & 1.67 & 0.09 & \\
\hline Weight (kg) & 62.9 & $8 \cdot 8$ & 63.4 & $9 \cdot 0$ & $63 \cdot 1$ & $9 \cdot 2$ & 63.7 & 9.5 & \\
\hline BMI $\left(\mathrm{kg} / \mathrm{m}^{2}\right)$ & $22 \cdot 6$ & 1.6 & $22 \cdot 7$ & 1.6 & $22 \cdot 6$ & 1.6 & $22 \cdot 8$ & 1.6 & \\
\hline Systolic BP & 120 & 6 & 117 & 7 & 117 & 9 & 118 & 9 & \\
\hline Diastolic BP & 76 & 7 & 73 & 8 & 75 & 10 & 73 & 10 & \\
\hline Pulse (beats per min) & 72 & 9 & 70 & 11 & 69 & 8 & 67 & 8 & \\
\hline $\mathrm{Hb}(\mathrm{g} / \mathrm{l})$ & 139 & 10 & 141 & 12 & 141 & 10 & 140 & 10 & $120-180$ \\
\hline Haematocrit (\%) & $41 \cdot 8$ & $2 \cdot 2$ & $42 \cdot 1$ & $2 \cdot 6$ & $42 \cdot 2$ & $2 \cdot 1$ & $42 \cdot 3$ & $2 \cdot 3$ & $40-54$ \\
\hline Erythrocytes $\left(\times 10^{12} /\right)$ & $4 \cdot 8$ & 0.3 & $4 \cdot 8$ & 0.4 & $4 \cdot 8$ & 0.4 & $4 \cdot 8$ & 0.3 & $3 \cdot 8-6 \cdot 5$ \\
\hline Leucocytes $\left(\times 10^{9} / \mathrm{I}\right)$ & 5.9 & 1.5 & $6 \cdot 1$ & 1.4 & $6 \cdot 3$ & $1 \cdot 2$ & $6 \cdot 3$ & 1.3 & $4.0-11.0$ \\
\hline Platelets $\left(\times 10^{9} / \mathrm{I}\right)$ & 239 & 52 & 240 & 52 & 238 & 55 & 238 & 49 & $150-400$ \\
\hline PDW (fl) & $13 \cdot 6$ & 1.8 & $14 \cdot 1$ & $2 \cdot 8$ & 14.0 & 1.9 & $13 \cdot 8$ & 1.9 & $9-14$ \\
\hline MPV (fl) & $11 \cdot 2$ & 0.8 & $11 \cdot 3$ & $1 \cdot 2$ & $11 \cdot 3$ & 0.8 & $11 \cdot 2$ & 0.8 & $7.5-11.5$ \\
\hline PT (s) & $11 \cdot 1$ & 0.5 & 10.9 & 0.8 & $11 \cdot 3$ & 0.6 & $10 \cdot 8$ & 0.6 & $11 \cdot 0-15 \cdot 0$ \\
\hline APTT (s) & $30 \cdot 2$ & 3.6 & $29 \cdot 2$ & $2 \cdot 2$ & $31 \cdot 3$ & $3 \cdot 1$ & $29 \cdot 3$ & $2 \cdot 3$ & $25 \cdot 0-35 \cdot 0$ \\
\hline Fibrinogen $(g / l)$ & $2 \cdot 41$ & 0.49 & $2 \cdot 50$ & 0.45 & $2 \cdot 48$ & 0.45 & 2.63 & 0.64 & $1 \cdot 5-4 \cdot 0$ \\
\hline TCT (s) & $17 \cdot 9$ & 1.6 & $18 \cdot 1$ & $1 \cdot 2$ & $17 \cdot 7$ & $1 \cdot 2$ & $18 \cdot 0$ & $1 \cdot 3$ & $<20$ \\
\hline D-Dimer (mg/l) & 0.29 & 0.38 & 0.36 & 0.60 & 0.31 & 0.37 & 0.30 & 0.42 & $<0.50$ \\
\hline
\end{tabular}

BP, blood pressure; PDW; platelet distribution width; MPV, mean platelet volume; PT, prothrombin time; APTT, activated partial thromboplastin time; TCT, thrombin clotting time.

Table 2. Distribution of volunteer micronutrient and macronutrient intake during supplementation bouts

(Mean values and standard deviations)

\begin{tabular}{|c|c|c|c|c|}
\hline \multirow[b]{2}{*}{ Parameters } & \multicolumn{2}{|c|}{ Supplementation bout 1} & \multicolumn{2}{|c|}{ Supplementation bout 2} \\
\hline & Mean & SD & Mean & SD \\
\hline Alcohol (g) & $16 \cdot 3$ & $44 \cdot 7$ & $13 \cdot 1$ & $45 \cdot 2$ \\
\hline$a$-Tocopherol (mg) & $10 \cdot 0$ & 4.2 & 8.9 & $4 \cdot 0$ \\
\hline Carbohydrates (g) & 195 & 79 & 194 & 69 \\
\hline Niacin (mg) & $27 \cdot 1$ & $12 \cdot 9$ & $25 \cdot 0$ & $13 \cdot 5$ \\
\hline Protein $(\mathrm{g})$ & 102 & 29 & 100 & 33 \\
\hline Riboflavin (mg) & $2 \cdot 3$ & $1 \cdot 1$ & $2 \cdot 3$ & 0.9 \\
\hline Sugars (g) & 93.6 & $47 \cdot 9$ & 93.0 & $44 \cdot 7$ \\
\hline Thiamin (mg) & $1 \cdot 6$ & $1 \cdot 1$ & 1.6 & $1 \cdot 2$ \\
\hline Total fat (g) & $78 \cdot 6$ & $26 \cdot 3$ & $70 \cdot 6$ & $23 \cdot 6$ \\
\hline Vitamin A $(\mu \mathrm{g})$ & 917 & 664 & 928 & 558 \\
\hline Vitamin $B_{12}(\mu \mathrm{g})$ & $6 \cdot 2$ & 3.2 & 5.9 & 2.9 \\
\hline Vitamin $B_{6}(\mathrm{mg})$ & 1.6 & 0.7 & 1.5 & 0.6 \\
\hline Vitamin C (mg) & $92 \cdot 6$ & $47 \cdot 8$ & $87 \cdot 9$ & $51 \cdot 5$ \\
\hline Vitamin E (mg) & $11 \cdot 1$ & 4.8 & $10 \cdot 1$ & 4.5 \\
\hline
\end{tabular}

\section{Platelet aggregation}

ADP-induced platelet aggregation was reduced by $29 \%$ (Fig. 5) post 4-week ACN supplementation. Arachidonic acid and collagen-induced platelet aggregation showed no significant changes post-ACN supplementation (data not shown). PBO supplementation did not affect platelet aggregation induced by any of the platelet agonists.

\section{Discussion}

The objective of this dietary intervention trial was to evaluate the effect of ACN supplementation on mechanistic pathways of thrombogenesis in sedentary pro-thrombotic population. It was observed that $320 \mathrm{mg} / \mathrm{d}$ of ACN capsule for 4 weeks had the

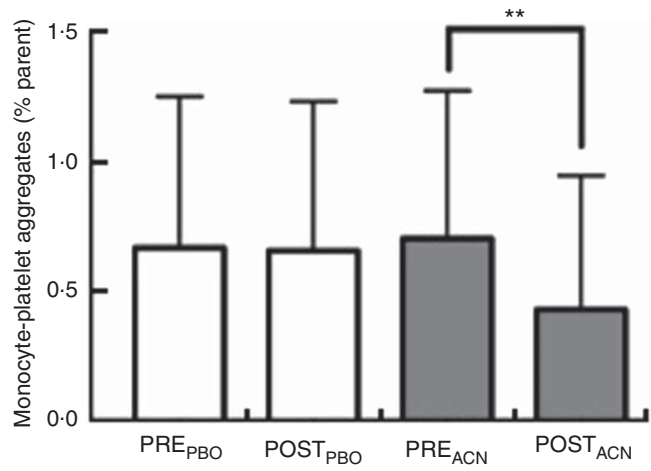

Fig. 1. Effect of anthocyanin (ACN) supplementation on monocyte-platelet aggregate formation using CD14/CD42b surface marker expression. ACN supplementation, 4 weeks, reduced monocyte-platelet aggregate formation by $39 \%(\downarrow 0.28$ (SD 0.19), $P=0.0079)$. Data are represented as supplementation type $v$. percentage parent population. Values are means $(n 16)$, and standard deviations represented by vertical bars. $\mathrm{PBO}$, placebo. ${ }^{*}$ Statistical significance $(P<0.01)$.

ability to alleviate both ADP-induced platelet activation and aggregation; biomarkers of thrombogenesis such as monocyteplatelet activation and PECAM-1 expression, demonstrating the potential to blunt a similar pathway targeted by current anti-platelet drugs such as clopidogrel.

Although there were no significant changes in full blood count, coagulation profile, biochemical profile or inflammatory markers post-ACN supplementation in this study, there have been other in vivo studies that have demonstrated diverse systemic effects. In a randomised, double-blind PBO-controlled dietary intervention trial of $200 \mathrm{ml} / \mathrm{d}$ Queen Garnet plum juice consumption for $28 \mathrm{~d}$ Santhakumar et al. demonstrated an increase in prothrombin time and a decrease in fibrinogen concentration $^{(1)}$. Another in vivo study evaluating the effect of blueberry supplementation on forty-eight obese men and 


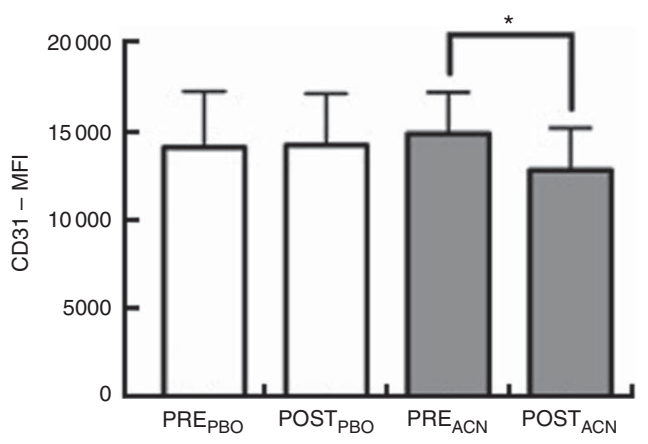

Fig. 2. Effect of anthocyanin (ACN) supplementation on CD31 (platelet endothelial cell adhesion molecule-1) surface marker expression. ACN supplementation, 4 weeks, reduced CD31 expression by $14 \%$ ( $\downarrow 2041$ (SD 832), $P=0.0202$ ). Data are represented as type of supplement $v$. mean fluorescence intensity (MFI). Values are means ( $n 16)$, and standard deviations represented by vertical bars. PBO, placebo. * Statistical significance $(P<0.05)$.

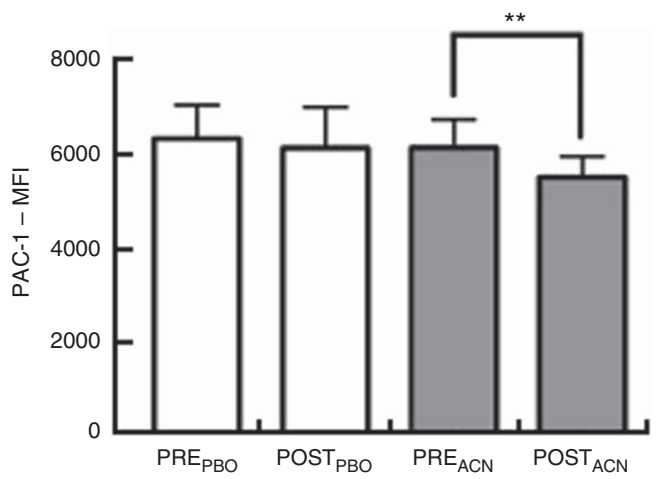

Fig. 3. Effect of anthocyanin (ACN) supplementation on procaspase activating compound-1 (PAC-1) expression. PAC-1 showed a decreased expression post 4week ACN supplementation by $10 \%$ ( $\downarrow 626$ (SD 188), $P=0.0023$ ). Data are represented as type of supplement $v$. mean fluorescence intensity (MFI). Values are means $(n 16)$, and standard deviations represented by vertical bars. PBO, placebo. ${ }^{* *}$ Statistical significance $(P<0.01)$.

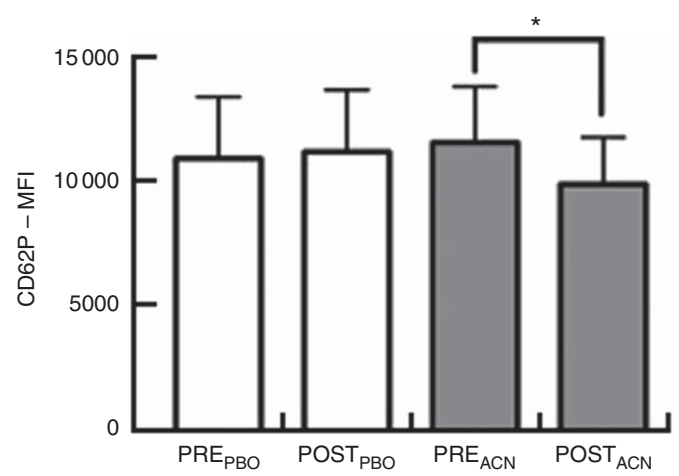

Fig. 4. Effect of anthocyanin (ACN) supplementation on CD62P (P-selectin). ACN supplementation, 4 weeks, reduced CD62P expression by $14 \%(\downarrow 1673$ (SD 723), $P=0.0285$ ). Data are represented as type of supplement $v$. mean fluorescence intensity (MFI). Values are means $(n 16)$, and standard deviations represented by vertical bars. PBO, placebo. ${ }^{*}$ Statistical significance $(P<0.05)$.

women with metabolic syndrome demonstrated no significant change in glucose and lipid profiles ${ }^{(20)}$. However, an in vivo study observing the effects of freeze-dried strawberry powder

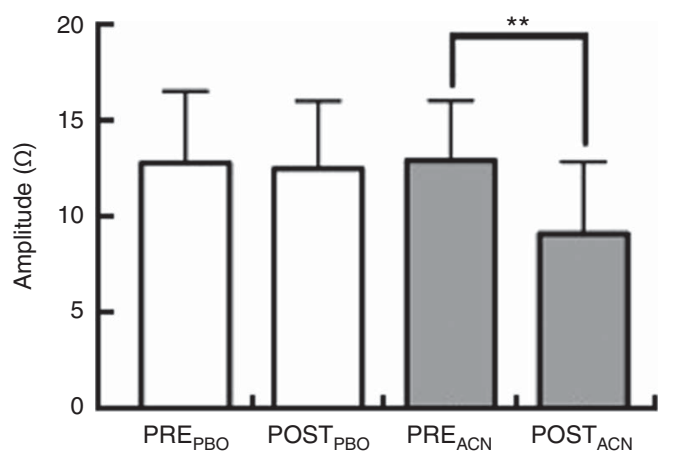

Fig. 5. Effect of 4-week anthocyanin (ACN) supplementation on ADP-induced whole blood platelet aggregation. $A C N$ supplementation reduced platelet aggregation by $29 \%(\downarrow 3.8$ (SD 1.2$), P=0.0039)$. Data are represented as type of supplementation $v$. amplitude $(\Omega)$. Values are means $(n 16)$, and standard deviations represented by vertical bars. PBO, placebo. ** Statistical significance $(P<0.01)$.

on sixteen females who presented with features of metabolic syndrome demonstrated a decrease in total cholesterol and LDL-cholesterol, but no significant changes to the inflammatory marker (C-reactive protein) level post 4-week supplementation $^{(27)}$. Furthermore, a decrease in LDL-cholesterol and increase in HDL-cholesterol concentrations were observed post $320 \mathrm{mg} / \mathrm{d}$ of ACN supplementation for 12 weeks in 120 dyslipidaemic subjects ${ }^{(28)}$. It can be deduced from previous studies focusing on various $\mathrm{ACN}$-rich supplementations that there have been divergent outcomes associated with haemostatic, biochemical and inflammatory markers ${ }^{(1,23,27)}$.

Biomarkers of thrombogenesis such as monocyte-platelet aggregate formation and PECAM-1 were inhibited post 4-week ACN supplementation. Monocyte-platelet aggregate formation has been established as a reliable marker of thrombus development ${ }^{(7)}$. This study demonstrated a decrease in monocyteplatelet aggregate formation by $39 \%(P<0 \cdot 01)$ post 4 -week ACN supplementation thereby exhibiting a potential role in reducing thrombus acceleration. Due to its ability to mediate leucocyte infiltration, PECAM-1 when highly expressed on leucocyte population has been linked with atherosclerotic involvement ${ }^{(29)}$. The decreased expression of PECAM- 1 in this study by $14 \% \quad(P<0.05)$ demonstrates ACN ability to reduce leucocyte migration and aggregate formation in the damaged endothelium that would typically occur during atherogenesis $^{(16)}$. In an ex vivo study evaluating the antithrombogenic properties of a phenolic metabolite, shikimic acid (SA), a reduced expression of PECAM-1 was observed posttreatment with $1 \mathrm{~mm}$ of SA, also demonstrating the ability of polyphenols in minimising thrombus growth ${ }^{(29)}$.

Platelet activation related conformational changes occur via the GPIIb/IIIa complex which reveals a ligand binding site specific for fibrinogen, von Willebrand factor, fibronectin and vitronectin $^{(30)}$. This binding site is vital for promoting platelet aggregation (secondary haemostatic response), which is recognised by PAC-1 and is exclusive for its ability and specificity to bind to only activated platelets ${ }^{(30)}$. In this current study, PAC-1 expression was inhibited by $10 \%(P<0.01)$ and therefore demonstrates ACN supplementation capacity to exhibit antiplatelet activation potential induced by ADP platelet 
activation receptor. An in vivo study performed on thirteen participants who consumed ACN-rich QGPJ with and without exercise-induced oxidative stress for $28 \mathrm{~d}$ demonstrated no significant change in PAC-1 expression ${ }^{(31)}$. In an ex vivo study focusing on hippuric acid and its ability to reduce activation related thrombogenesis also showed a reduction in PAC-1 expression $^{(13)}$. During platelet activation by agonists such as ADP, collagen or arachidonic acid in vivo, platelets release $\alpha$-granules. These secretory granules then translocate to the plasma membrane of endothelial cells, playing a crucial role in leucocyte recruitment to the site of injury ${ }^{(29)}$. In this study involving pro-thrombotic sedentary population, P-selectin expression was reduced by $14 \%(P<0.05)$, hence alleviating platelet activation related degranulation and subsequently blunting platelet-fibrin/ platelet-platelet binding (aggregation). In an in vitro study involving delphinidin-3-glucoside on three healthy volunteers, P-selectin and PAC-1 expression were also reduced $^{(32)}$. Another in vitro study observing the effects of black soyabean extracts on healthy males also demonstrated a reduction in P-selectin expression ${ }^{(33)}$. Whole blood ADP-induced platelet aggregation was also notably reduced post-ACN supplementation in this study by $29 \%(P<0 \cdot 01)$. Current antiplatelet therapeutics such as clopidogrel, ticagrelor and prasugrel target the $\mathrm{P}_{2} \mathrm{Y}_{1} / \mathrm{P}_{2} \mathrm{Y}_{12}$ pathways of platelet activation and aggregation $^{(12,34)}$. But these antiplatelet therapeutics have been associated with increased bleeding risk and resistance in pro-thrombotic populations ${ }^{(12,35)}$. The anti-radical and anti-thrombotic activity of ACN is attributable to its B benzoyl ring structure (delphinins and cyanidins), the hydroxylation, methoxylation and the $O$-diphenyl structure of the B-ring have been ascribed to the blunting of $\alpha \operatorname{IIb} \beta 3$ integrin activation and blocking of the ADP receptor $\left(\mathrm{P}_{2} \mathrm{Y}_{1} / \mathrm{P}_{2} \mathrm{Y}_{12}\right)$ platelet activation pathway ${ }^{(32)}$. The ability of the B-ring structure to attenuate these pathways has been exhibited in this study via reduction of ADP-induced platelet aggregation and platelet degranulation and therefore allowing ACNs to target similar pathways as currently used anti-platelet drugs ${ }^{(36)}$.

This study showed no significant reduction in collagen or arachidonic acid induced platelet aggregation, indicating ACN supplementation in this study did not blunt the GPVI or COX-1 pathway of platelet activation in the sedentary population. An in vivo study evaluating the effects of ACNs on platelet function also demonstrated no reactivity to collagen-induced platelet activation $^{(37)}$. Alternatively, in a recently conducted in vivo study by our group on overweight and obese participants, 4-week ACN supplementation showed inhibition of collagen and arachidonic acid stimulated platelet aggregation ${ }^{(38)}$. Furthermore, ACN-rich food supplements have exhibited a greater arachidonic acid platelet aggregation inhibitory effect during a simulated physiological oxidative stress environment ${ }^{(26)}$. It is believed that ACN intervention has the potential to inhibit prothrombotic pathways triggered by collagen and arachidonic acid (COX-pathway) greater under oxidative stress environments such as in obese/overweight or diabetic population $^{(38)}$. Although platelet aggregation has been an effective measure of thrombotic risk in humans, reproducibility and the need for repetitive standardisation should be taken into consideration. Nevertheless, there was minimal variability (tests performed in duplicates) observed in this study and in previous studies performed by our group ${ }^{(38)}$.

\section{Conclusion}

It was observed that $\mathrm{ACN}$ supplementation for 4 weeks reduces ADP-induced platelet activation, aggregation and biomarkers of thrombogenesis such as monocyte-platelet aggregate formation and PECAM-1 expression. Owing to a similar platelet activation receptor blunting effect as exhibited by current antiplatelet therapy, it is suggested that ACN could potentially act as a natural complementary therapeutic in pro-thrombotic sedentary population. Further mechanistic studies comparing intervention of clopidogrel $v$. ACN in pro-thrombotic population is warranted.

\section{Acknowledgements}

The authors would like to sincerely thank QML Pathology and Central Queensland University for their resources. A special mention to Mr Mitchell Thorpe, Ms Alyssa Tyrrell and Mrs Judy Couper for their technical assistance.

This clinical trial was financially supported by the Central Queensland University New Staff Grant, Queensland, Australia.

K. T. performed experimental analysis and prepared the manuscript. H. H. performed experimental analysis. W. P. and I. S. assisted in experimental design and manuscript critical review. A. B. S. designed the study, analysed data and critically reviewed the manuscript.

None of the authors has any conflicts of interest to declare.

\section{Supplementary material}

For supplementary material/s referred to in this article, please visit https://doi.org/10.1017/S0007114517002124

\section{References}

1. Santhakumar AB, Kundur A, Fanning K, et al. (2015) Consumption of anthocyanin-rich Queen Garnet plum juice reduces platelet activation related thrombogenesis in healthy volunteers. J Funct Foods 12, 11-22.

2. Laufs U, Wassmann S, Czech T, et al. (2005) Physical inactivity increases oxidative stress, endothelial dysfunction, and atherosclerosis. Arterioscler Thromb Vasc Biol 25, 809-814.

3. Pate RR, O'Neill JR \& Lobelo F (2008) The evolving definition of 'sedentary'. Exerc Sport Sci Rev 36, 173-178.

4. Chevion S, Moran DS, Heled Y, et al. (2003) Plasma antioxidant status and cell injury after severe physical exercise. Proc Natl Acad Sci U S A 100, 5119-5123.

5. Harrison P (2005) Platelet function analysis. Blood Rev 19, 111-123.

6. Gachet C (2015) Antiplatelet drugs: which targets for which treatments? J Thromb Haemost 13, Suppl 1, S313-S322.

7. Freedman JE (2002) Platelet-monocyte aggregates: bridging thrombosis and inflammation. Circulation 105, 2130-2132.

8. Michelson AD, Barnard MR, Krueger LA, et al. (2001) Circulating monocyte-platelet aggregates are a more sensitive marker of in vivo platelet activation than platelet surface P-selectin: studies in baboons, human coronary intervention, and human acute myocardial infarction. Circulation 104, $1533-1537$ 
9. Ford ES \& Caspersen CJ (2012) Sedentary behaviour and cardiovascular disease: a review of prospective studies. Int J Epidemiol 41, 1338-1353.

10. Ferreiro JL \& Angiolillo DJ (2011) Diabetes and antiplatelet therapy in acute coronary syndrome. Circulation 123, 798-813.

11. Roth G \& Calverley D (1994) Aspirin, platelets, and thrombosis: theory and practice. Blood $\mathbf{8 3}, 885-898$.

12. Thompson K, Pederick W \& Santhakumar AB (2016) Anthocyanins in obesity-associated thrombogenesis: a review of the potential mechanism of action. Food Funct 7, 2169-2178.

13. Santhakumar AB, Stanley R \& Singh I (2015) The ex vivo antiplatelet activation potential of fruit phenolic metabolite hippuric acid. Food Funct 6, 2679-2683.

14. Alvarez-Suarez JM, Giampieri F, Tulipani S, et al. (2014) One-month strawberry-rich anthocyanin supplementation ameliorates cardiovascular risk, oxidative stress markers and platelet activation in humans. J Nutr Biochem 25, 289-294.

15. Benn T, Kim B, Park YK, et al. (2015) Polyphenol-rich blackcurrant extract exerts hypocholesterolaemic and hypoglycaemic effects in mice fed a diet containing high fat and cholesterol. Br J Nutr 113, 1697-1703.

16. Chong MF, Macdonald R \& Lovegrove JA (2010) Fruit polyphenols and CVD risk: a review of human intervention studies. Br J Nutr 104, Suppl. 3, S28-S39.

17. Santhakumar AB, Bulmer AC \& Singh I (2014) A review of the mechanisms and effectiveness of dietary polyphenols in reducing oxidative stress and thrombotic risk. J Hum Nutr Diet 27, 1-21.

18. Dembinska-Kiec A, Mykkanen O, Kiec-Wilk B, et al. (2008) Antioxidant phytochemicals against type 2 diabetes. Br J Nutr 99, E Suppl. 1, ES109-ES117.

19. Amiot MJ, Riva C \& Vinet A (2016) Effects of dietary polyphenols on metabolic syndrome features in humans: a systematic review. Obes Rev 17, 573-586.

20. Basu A, Du M, Leyva MJ, et al. (2010) Blueberries decrease cardiovascular risk factors in obese men and women with metabolic syndrome. J Nutr 140, 1582-1587.

21. Bell DR \& Gochenaur K (2006) Direct vasoactive and vasoprotective properties of anthocyanin-rich extracts. $J$ Appl Physiol (1985) 100, 1164-1170.

22. Edirisinghe I, Banaszewski K, Cappozzo J, et al. (2011) Strawberry anthocyanin and its association with postprandial inflammation and insulin. Br J Nutr 106, 913-922.

23. Farrell NJ, Norris GH, Ryan J, et al. (2015) Black elderberry extract attenuates inflammation and metabolic dysfunction in diet-induced obese mice. Br J Nutr 114, 1123-1131.

24. Manach C, Williamson G, Morand C, et al. (2005) Bioavailability and bioefficacy of polyphenols in humans. I. Review of 97 bioavailability studies. Am J Clin Nutr 81, 230S-242S.
25. Santhakumar AB, Kundur AR, Fanning K, et al. (2015) Consumption of anthocyanin-rich Queen Garnet plum juice reduces platelet activation related thrombogenesis in healthy volunteers. J Funct Foods 12, 11-22.

26. Royal College of Pathologists of Australasia (2004) RCPA Manual. Surry Hills, NSW: RCPA.

27. Basu A, Wilkinson M, Penugonda K, et al. (2009) Freeze-dried strawberry powder improves lipid profile and lipid peroxidation in women with metabolic syndrome: baseline and post intervention effects. Nutr $J \mathbf{8}, 43$.

28. Qin Y, Xia M, Ma J, et al. (2009) Anthocyanin supplementation improves serum LDL- and HDL- cholesterol concentrations associated with the inhibition of cholesteryl ester transfer protein in dyslipidemic subjects. Am J Clin Nutr $\mathbf{0}, 1-8$

29. Veach D, Hosking H, Thompson K, et al. (2016) Anti-platelet and anti-thrombogenic effects of shikimic acid in sedentary population. Food Funct 7, 3609-3616.

30. Calvete JJ (1995) On the structure and function of platelet integrin alpha IIb beta 3, the fibrinogen receptor. Proc Soc Exp Biol Med 208, 346-360.

31. Santhakumar AB, Kundur A, Sabapathy S, et al. (2015) The potential of anthocyanin-rich Queen Garnet plum juice supplementation in alleviating thrombotic risk under induced oxidative stress conditions. J Funct Foods $\mathbf{1 4}$ 747-757.

32. Yang Y, Shi Z, Reheman A, et al. (2012) Plant food delphinidin3-glucoside significantly inhibits platelet activation and thrombosis: novel protective roles against cardiovascular diseases. PLOS ONE 7, e37323.

33. Kim K, Lim KM, Kim CW, et al. (2011) Black soybean extract can attenuate thrombosis through inhibition of collagen-induced platelet activation. J Nutr Biochem 22, 964-970.

34. Becker RC, Bassand JP, Budaj A, et al. (2011) Bleeding complications with the P2Y12 receptor antagonists clopidogrel and ticagrelor in the PLATelet inhibition and patient Outcomes (PLATO) trial. Eur Heart J 32, 2933-2944.

35. Bhatt DL (2008) What makes platelets angry: diabetes, fibrinogen, obesity, and impaired response to antiplatelet therapy? J Am Coll Cardiol 52, 1060-1061.

36. Fernandes I, Faria A, Calhau C, et al. (2014) Bioavailability of anthocyanins and derivatives. J Funct Foods 7, 54-66.

37. Rechner AR \& Kroner C (2005) Anthocyanins and colonic metabolites of dietary polyphenols inhibit platelet function. Thromb Res 116, 327-334.

38. Thompson K, Pederick W, et al. (2017) Anthocyanin supplementation in alleviating thrombogenesis in overweight and obese population: a randomized, double-blind, placebocontrolled study. J Funct Foods 32, 131-138. 